Article

\title{
Synthesis and Anticancer Activity of Some Novel Tetralin-6-yl-pyrazoline, 2-Thioxopyrimidine, 2-Oxopyridine, 2-Thioxo-pyridine and 2-Iminopyridine Derivatives
}

\section{Ebtehal S. Al-Abdullah}

Department of Pharmaceutical Chemistry, College of Pharmacy, King Saud University, Riyadh 11451, Saudi Arabia; E-Mail: ealabdullah@ksu.edu.sa

Received: 7 March 2011; in revised form: 29 March 2011 / Accepted: 8 April 2011 /

Published: 20 April 2011

\begin{abstract}
The title compounds were prepared by reaction of 6-acetyltetralin (1) with different aromatic aldehydes 2a-c, namely 2,6-dichlorobenzaldehyde, 2,6-diflourobenzaldehyde, and 3-ethoxy-4-hydroxybenzaldehyde, to yield the corresponding $\alpha, \beta$-unsaturated ketones 3a-c. Compound $\mathbf{3 b}$ was reacted with hydrazine hydrate to yield the corresponding 2-pyrazoline $\mathbf{4}$, while compounds $\mathbf{3 a , b}$ reacted with thiourea to afford the 2-thioxopyrimidine derivatives $\mathbf{5 a}, \mathbf{b}$, respectively. The reaction of $\mathbf{1}$, and the aromatic aldehydes 2a-c with ethyl cyanoacetate, 2-cyano-thioacetamide or malononitrile in the presence of ammonium acetate yielded the corresponding 2-oxopyridines $\mathbf{6 a}, \mathbf{b}$, 2-thioxopyridines $\mathbf{7 a - c}$ or 2-iminopyridines $\mathbf{8 a}, \mathbf{b}$, respectively. The newly prepared compounds were evaluated for anticancer activity against two human tumor cell lines. Compound 3a showed the highest potency with $\mathrm{IC}_{50}=3.5$ and $4.5 \mu \mathrm{g} / \mathrm{mL}$ against a cervix carcinoma cell line (Hela) and breast carcinoma cell line (MCF7), respectively.
\end{abstract}

Keywords: tetrahydronaphthalenes; 2-pyrazoline; 2-thioxopyrimidine; 2-oxopyridine; 2-thioxopyridine; 2-iminipyridine; anticancer activity

\section{Introduction}

Cancer is presently responsible for about $25 \%$ of deaths in developed countries and for $15 \%$ of all deaths worldwide. It can therefore be considered as one of the foremost health problems, with about 1.45 million new cancer cases expected yearly. Antitumor chemotherapy is nowadays a very active field of research, and a huge amount of information on the topic is generated every year [1,2]. 
Although there is a large amount of information available dealing with clinical aspects of cancer chemotherapy, we felt that there was a clear need for an updated treatment from the point of view of medicinal chemistry and drug design [3]. Diverse chemotherapeutic activities were ascribed to substituted tetralin (tetrahydronaphthalene)-heterocycles [4,5] and tetrahydronaphthalene derivatives, especially those incorporated into heterocyclic systems [6]. It has been reported that this type of compounds possess a wide variety of biological activities including anti-HIV [5], antibacterial [4,7], hypotensive [8], antiarrythmic [9], molluscicidal [10], antiplatelet aggregation [11,12], anxiolytic and antidepressant $[13,14]$ and anticancer $[15,16]$ effects. Pyridin-2 $(1 H)$-ones are known to possess a range of biological activities such as analgesic, antifungal, antimalarial, antiinflammatory, antibacterial, anti-HIV, phytotoxic, antitumoral and antiviral properties [17-24]. Thus, it was of interest to synthesize some new heterocyclic derivatives carrying a tetrahydronaphthale moiety for evaluation as potential anticancer agents.

\section{Results and Discussion}

\subsection{Chemistry}

$\alpha, \beta$-Unsaturated ketons (chalcones) are active intermediates and excellent starting materials for the synthesis of several heterocyclic systems. Thus, a Claisen-Schmidt reaction of 1-(1,2,3,4-tetrahydronaphthaline-6-yl)ethanone (1), prepared following the previously reported method of Allinger and Jones [25], with 2,6-dichlorobenzaldehyde (2a), 2,6-diflourobenzaldehyde (2b) or 3-ethoxy-4-hydroxybenzaldehyde (2c), in 10\% ethanolic sodium hydroxide afforded the corresponding $\alpha, \beta$-unsaturated ketons 3a-c in 53, 42 and $80 \%$ yields, respectively. Condensation of the chalcone analogue $\mathbf{3 b}$ with hydrazine hydrate in acetic acid yielded the corresponding 1-acetyl-2-pyrazoline derivative 4 in 92\% yield. Meanwhile, the interaction of the chalcone analogues 3a,b with thiourea in aqueous potassium hydroxide yielded the corresponding 2-thioxopyrimidines 5a,b in 20 and 28\% yields, respectively (Scheme 1, Table 1).

Scheme 1. Synthetic pathways to compounds 3a-c, 4 and 5a,b.

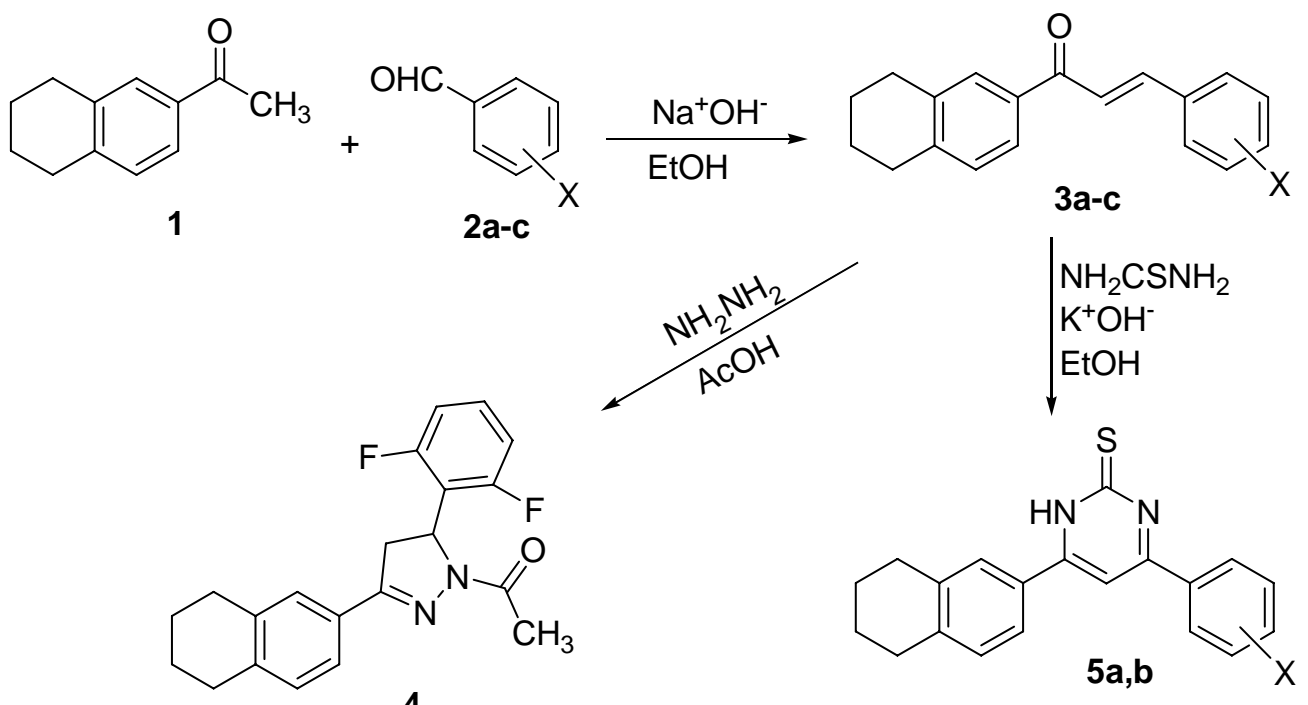


Table 1. Melting points, crystallization solvents, yields, molecular formulae and molecular weights of newly synthesized compounds $\mathbf{3 a - c}, \mathbf{4}, \mathbf{5 a}, \mathbf{b}, \mathbf{6 a}, \mathbf{b}, 7 \mathbf{7}-\mathbf{c}$ and $\mathbf{8 a}, \mathbf{b}$.

\begin{tabular}{cccccc}
\hline $\begin{array}{c}\text { Comp. } \\
\text { No. }\end{array}$ & $\mathbf{X}$ & $\mathbf{M p}\left({ }^{\mathbf{o}} \mathbf{C}\right)$ & Cryst. Solv. & $\begin{array}{c}\text { Yield } \\
(\mathbf{\%})\end{array}$ & $\begin{array}{c}\text { Molecular Formula } \\
\text { (Mol. Wt.) }\end{array}$ \\
\hline $\mathbf{3 a}$ & $2,6-\mathrm{Cl}_{2}$ & $71-3$ & $\mathrm{EtOH}$ & 53 & $\mathrm{C}_{19} \mathrm{H}_{16} \mathrm{Cl}_{2} \mathrm{O}(331.24)$ \\
$\mathbf{3 b}$ & $2,6-\mathrm{F}_{2}$ & $250-2$ & $\mathrm{EtOH}$ & 42 & $\mathrm{C}_{19} \mathrm{H}_{16} \mathrm{~F}_{2} \mathrm{O}(298.34)$ \\
$\mathbf{3 c}$ & $3-\mathrm{EtO}, 4-\mathrm{OH}$ & $77-9$ & $\mathrm{EtOH}$ & 80 & $\mathrm{C}_{20} \mathrm{H}_{20} \mathrm{O}_{2}(292.38)$ \\
$\mathbf{4}$ & - & $>300$ & $\mathrm{AcOH}$ & 95 & $\mathrm{C}_{21} \mathrm{H}_{20} \mathrm{~F}_{2} \mathrm{~N}_{2} \mathrm{O}(354.40)$ \\
$\mathbf{5 a}$ & $2,6-\mathrm{Cl}_{2}$ & $>300$ & $\mathrm{EtOH} / \mathrm{H}_{2} \mathrm{O}$ & 20 & $\mathrm{C}_{20} \mathrm{H}_{16} \mathrm{Cl}_{2} \mathrm{~N}_{2} \mathrm{~S}(387.33)$ \\
$\mathbf{5 b}$ & $2,6-\mathrm{F}_{2}$ & $253-5$ & $\mathrm{EtOH} / \mathrm{H}_{2} \mathrm{O}$ & 28 & $\mathrm{C}_{20} \mathrm{H}_{16} \mathrm{~F}_{2} \mathrm{~N}_{2} \mathrm{~S}(354.42)$ \\
$\mathbf{6 a}$ & $2,6-\mathrm{Cl}_{2}$ & $252-4$ & $\mathrm{AcOH}$ & 18 & $\mathrm{C}_{22} \mathrm{H}_{16} \mathrm{Cl}_{2} \mathrm{~N}_{2} \mathrm{O}(395.29)$ \\
$\mathbf{6 b}$ & $2,6-\mathrm{F}_{2}$ & $250-2$ & $\mathrm{AcOH}$ & 30 & $\mathrm{C}_{22} \mathrm{H}_{16} \mathrm{~F}_{2} \mathrm{~N}_{2} \mathrm{O}(362.38)$ \\
$7 \mathbf{7 a}$ & $2,6-\mathrm{Cl}_{2}$ & $150-2$ & $\mathrm{AcOH}$ & 25 & $\mathrm{C}_{22} \mathrm{H}_{16} \mathrm{Cl}_{2} \mathrm{~N}_{2} \mathrm{~S}(411.36)$ \\
$\mathbf{7 b}$ & $2,6-\mathrm{F}_{2}$ & $124-6$ & $\mathrm{AcOH}$ & 53 & $\mathrm{C}_{22} \mathrm{H}_{16} \mathrm{~F}_{2} \mathrm{~N}_{2} \mathrm{~S}(378.45)$ \\
$\mathbf{7 c}$ & $3-\mathrm{EtO}-4-\mathrm{OH}$ & $226-8$ & $\mathrm{AcOH}$ & 90 & $\mathrm{C}_{23} \mathrm{H}_{20} \mathrm{~N}_{2} \mathrm{OS}(372.49)$ \\
$\mathbf{8 a}$ & $2,6-\mathrm{Cl}_{2}$ & $193-5$ & $\mathrm{AcOH}$ & 26 & $\mathrm{C}_{22} \mathrm{H}_{17} \mathrm{Cl}_{2} \mathrm{~N}_{3}(394.31)$ \\
$\mathbf{8 b}$ & $2,6-\mathrm{F}_{2}$ & $100-2$ & $\mathrm{AcOH}$ & 34 & $\mathrm{C}_{22} \mathrm{H}_{17} \mathrm{~F}_{2} \mathrm{~N}_{3}(361.40)$ \\
\hline
\end{tabular}

Condensation of compound $\mathbf{1}$ and the aromatic aldehydes $\mathbf{2 a}$ or $\mathbf{2} \mathbf{b}$ with ethyl cyanoacetate in presence of excess ammonium acetate in $n$-butanol afforded the corresponding 3-cyano-2-(1H)oxopyridines $6 \mathbf{a}$ and $\mathbf{6 b}$ in a one pot reaction. Similarly, the reaction of compound $\mathbf{1}$ and the aromatic aldehydes 2a-c with 2-cyanothioacetamide yielded the corresponding 3-cyano-2-(1H)-thioxopyridines 7a-c in 25, 53 and 90\% yields, respectively. The 3-cyano-2-(1H)-iminopyridines 8a and $\mathbf{8 b}$ were also prepared in fair yields by applying the aforementioned one pot reaction of the compound $\mathbf{1}$, the corresponding aldehyde and malononitrile (Scheme 2, Table 1). The structures of the newly synthesized compounds were confirmed on the basis of their elemental analysis and IR, ${ }^{1} \mathrm{H}-\mathrm{NMR}$, ${ }^{13} \mathrm{C}-\mathrm{NMR}$ and mass spectral data.

Scheme 2. Synthetic pathways of compounds $\mathbf{6 a}, \mathbf{b}, 7 \mathbf{a}-\mathbf{c}$ and $\mathbf{8 a}, \mathbf{b}$.

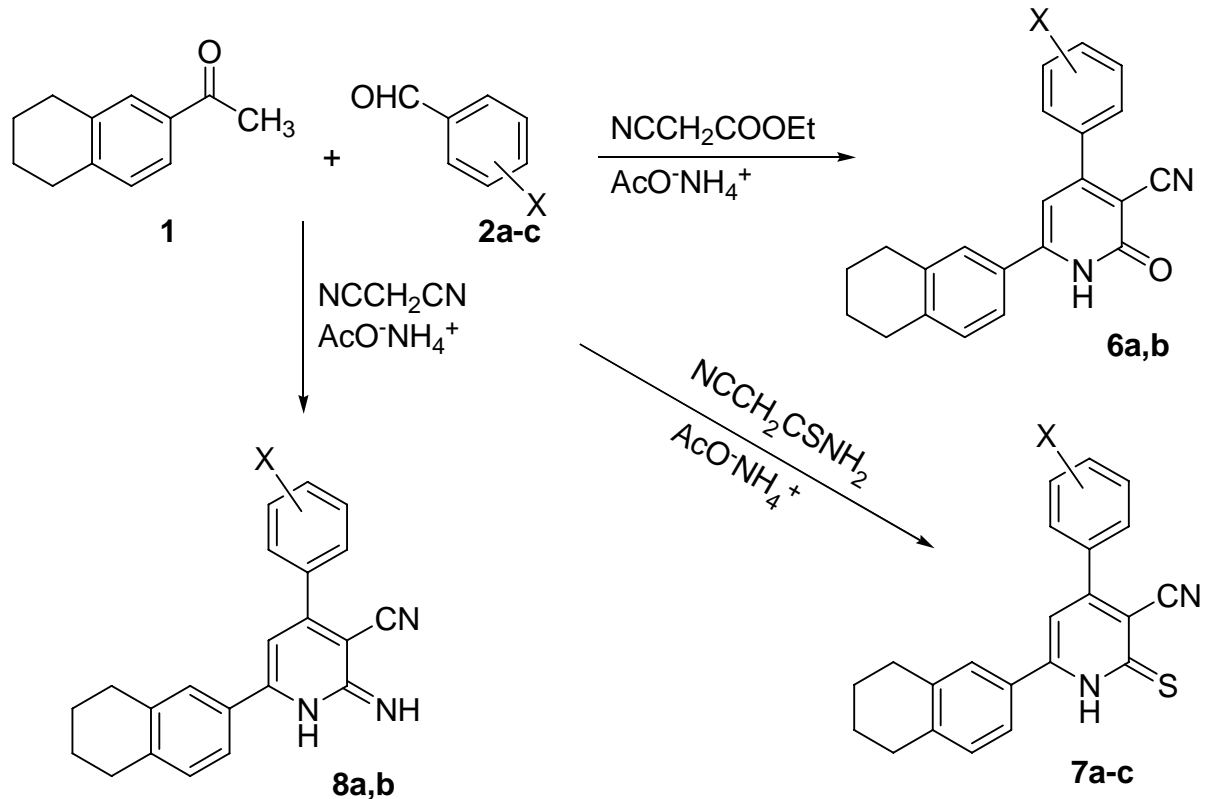




\subsection{Anticancer Screening}

All the newly synthesized compounds were tested at the Department of Tumor Pathology, National Cancer Institute, Cairo, Egypt. Two cell lines were used for the evaluation - human cervix carcinoma cell line (Hela) and human breast carcinoma cell line (MCF7). The newly synthesized analogs 3a-c, 4, $\mathbf{5 a}, \mathbf{b}, \mathbf{6 a}, \mathbf{b}, \mathbf{7} \mathbf{a}-\mathbf{c}$ and $\mathbf{8 a}, \mathbf{b}$ were tested for in vitro cytotoxic activity against these cell lines, which were obtained frozen in liquid nitrogen $\left(-180{ }^{\circ} \mathrm{C}\right)$ from the American Type Culture Collection [26]. The tumor cell lines were maintained in the National Cancer Institute, Cairo, Egypt, by serial sub-culturing.

The results (Table 2) are expressed in the form of the concentration of compound that causes $50 \%$ inhibition of cells growth. The in vitro evaluation revealed that all the newly synthesized compounds showed certain activity against tumor cell lines tested, although the activity was generally higher towards the cervical cancer line than the breast cancer one. Compound 3a showed potent and broad antitumor activity against the two tumor cell lines tested $\left(\mathrm{IC}_{50}=3.5\right.$ against Hela and $\mathrm{IC}_{50}=4.5$ against MCF7) compared to the potent anticancer drug 5-flourouracil (5-FU) used as a reference standard [27]. Substitution of the side chain with different ring systems like in the oxopyridine or thioxopyridine compounds $\mathbf{6 a}, \mathbf{b}$ and $7 \mathbf{a}, \mathbf{b}$ resulted in moderate activity against the Hela cell line and marked activity against the MCF-7 cell line. Meanwhile, substitution of the side chain with iminopyridine, thioxopyrimidine and/or pyrazoline rings resulted in low activity against the two tumor cell lines. These results demonstrated that changing the molecular conformation and orientation could influence markedly the antitumor activity against the two tested cell types.

Table 2. Effect of compounds 3a-c, 4, 5a, $\mathbf{b}, \mathbf{6 a}, \mathbf{b}, 7 \mathbf{a}-\mathbf{c}$ and $\mathbf{8 a}, \mathbf{b}$ on cervix carcinoma cell line (Hela) and breast carcinoma cell line (MCF7).

\begin{tabular}{ccc}
\hline \multirow{2}{*}{ Compound No. } & \multicolumn{2}{c}{$\mathbf{I C}_{\mathbf{5 0}}(\boldsymbol{\mu g} / \mathbf{m L})$} \\
\cline { 2 - 3 } 3a & 3.5 & MCF7 \\
\hline $\mathbf{3 b}$ & 10.5 & 4.5 \\
$\mathbf{3 c}$ & 12.5 & 15 \\
$\mathbf{4}$ & 11.3 & 18.3 \\
$\mathbf{5 a}$ & 10.7 & 19 \\
$\mathbf{5 b}$ & 11.9 & 20.5 \\
$\mathbf{6 a}$ & 7.1 & 17.3 \\
$\mathbf{6 b}$ & 10.9 & 12 \\
$\mathbf{7 a}$ & 8.1 & 17.5 \\
$\mathbf{7 b}$ & 5.9 & 16 \\
$\mathbf{7} \mathbf{c}$ & 6.5 & 12.5 \\
$\mathbf{8 a}$ & 12.1 & 16 \\
$\mathbf{8 b}$ & 12.1 & 22.3 \\
$\mathbf{5 - F U}$ & - & 21.7 \\
\hline
\end{tabular}




\section{Experimental}

\subsection{General}

Melting points $\left({ }^{\circ} \mathrm{C}\right.$, uncorrected) were measured in open glass capillaries using a Barnstead 9001 electrothermal melting point apparatus. Infrared spectra $\left(v, \mathrm{~cm}^{-1}\right)$ were recorded on a Jasco FT/IR-330E, Fourier Transform Infrared Spectrometer using $\mathrm{KBr}$ discs. ${ }^{1} \mathrm{H}-\mathrm{NMR}$ spectra were determined using a Bruker AC 500 Ultra Shield NMR spectrometer operating at $500.13 \mathrm{MHz}$ for ${ }^{1} \mathrm{H}$ and $125.76 \mathrm{MHz}$ for ${ }^{13} \mathrm{C}$, the chemical shifts are expressed in $\delta$ (ppm) downfield from tetramethylsilane (TMS) used as internal standard. Electron impact mass spectra (EI-MS) were recorded on a Shimadzu GC-MS-QP 5000 instrument at $70 \mathrm{eV}$. Elemental analyses (C, H, N) were in full agreement with the proposed structures within $\pm 0.4 \%$ of the theoretical values. Monitoring the reactions and checking the purity of the final products were carried out by thin layer chromatography (TLC) using silica gel precoated aluminum sheets $\left(60 \mathrm{~F}_{254}\right.$, Merck) and visualization with ultraviolet light (UV) at 365 and $254 \mathrm{~nm}$.

\subsection{3-Aryl-1-(1,2,3,4-tetrahydronaphthalen-6-yl)prop-2-en-1-ones 3a-c}

A mixture of 6-acetyltetraline $1(4.9 \mathrm{~g}, 0.028 \mathrm{~mol})$, the appropriate aldehyde $\mathbf{2 a}-\mathbf{c}(0.028 \mathrm{~mol})$ and $10 \%$ ethanolic sodium hydroxide solution $(15 \mathrm{~mL})$ in ethanol $(30 \mathrm{~mL})$ was stirred for $12 \mathrm{~h}$. The reaction mixture was then warmed at $40{ }^{\circ} \mathrm{C}$ for $10 \mathrm{~min}$. and the separated precipitate was filtered off and recrystallized from ethanol.

3-(2,6-Dichlorophenyl)-1-(1,2,3,4-tetrahydronaphthalen-6-yl)prop-2-en-1-ones (3a): IR: $1690(\mathrm{C}=\mathrm{O})$, $1650(\mathrm{C}=\mathrm{C}) .{ }^{1} \mathrm{H}-\mathrm{NMR}\left(\mathrm{DMSO}-\mathrm{d}_{6}\right): \delta 1.7-1.73\left(\mathrm{~m}, 4 \mathrm{H}\right.$, tetralin $\left.\mathrm{CH}_{2}\right), 2.76-2.8\left(\mathrm{~m}, 4 \mathrm{H}\right.$, tetralin $\left.\mathrm{CH}_{2}\right)$, $7.21(\mathrm{~d}, 1 \mathrm{H}, \mathrm{CH}=, J=10.4 \mathrm{~Hz}), 7.54(\mathrm{~d}, 1 \mathrm{H}, \mathrm{CH}=, J=10.4 \mathrm{~Hz}), 7.37-7.8(\mathrm{~m}, 6 \mathrm{H}, \mathrm{Ar}-\mathrm{H}) .{ }^{13} \mathrm{C}-\mathrm{NMR}: \delta$ 22 (tetralin $\mathrm{CH}_{2}$ ), 29 (tetralin $\mathrm{CH}_{2}$ ), $125.5(\mathrm{CH}=)$ ), $143.2(\mathrm{CH}=)$, 129.01, 129.25, 130.59, 130.83, 132.11, 134.37, 134.3, 135.07, 136.2, 137.3 (Ar-C), $188.5(\mathrm{C}=\mathrm{O}) . \mathrm{MS}, \mathrm{m} / \mathrm{z}$ (rel. int.): $334\left(\mathrm{M}^{+}, 0.3\right)$, $176(50), 175$ (75), 173 (100), $138(5)$.

3-(2,6-Difluorophenyl)-1-(1,2,3,4-tetrahydronaphthalen-6-yl)prop-2-en-1-ones (3b): IR: $1651(\mathrm{C}=\mathrm{O})$, $1548(\mathrm{C}=\mathrm{C}),{ }^{1} \mathrm{H}-\mathrm{NMR}\left(\mathrm{DMSO}_{\mathrm{d}}\right): \delta 1.7\left(\mathrm{~m}, 4 \mathrm{H}\right.$, tetralin $\left.\mathrm{CH}_{2}\right), 2.5\left(\mathrm{~m}, 4 \mathrm{H}\right.$, tetralin $\left.\mathrm{CH}_{2}\right), 7.24(\mathrm{~d}, 1 \mathrm{H}$, $\mathrm{CH}=, J=8.3 \mathrm{~Hz}), 7.84(\mathrm{~d}, 1 \mathrm{H}, \mathrm{CH}=, J=8.3 \mathrm{~Hz}), 6.66-7.6(\mathrm{~m}, 6 \mathrm{H}, \mathrm{Ar}-\mathrm{H}) ;{ }^{13} \mathrm{C}-\mathrm{NMR}: \delta 22$ (tetralin $\left.\mathrm{CH}_{2}\right), 29$ (tetralin $\left.\mathrm{CH}_{2}\right), 129.3(\mathrm{CH}=), 136.6(\mathrm{CH}=), 111.2,112.3,126.7,129.9,131.16,134.26,134.3$, 135.9, 142.5, 157.3 (Ar-C), $186.9(\mathrm{C}=\mathrm{O}) . \mathrm{MS}, \mathrm{m} / \mathrm{z}$ (rel. int.): $298\left(\mathrm{M}^{+}, 0.2\right), 165$ (19), 139 (2), 137 (100), $131(3), 75(5)$.

3-(3-Ethoxy-4-hydroxyphenyl))-1-(1,2,3,4-tetrahydronaphthalen-6-yl)prop-2-en-1-ones (3c): IR: 3420 $(\mathrm{OH}), 1634(\mathrm{C}=\mathrm{O}) 1539(\mathrm{C}=\mathrm{C}) .{ }^{1} \mathrm{H}-\mathrm{NMR}: \delta 1.35$ (t, 3H, $\left.\mathrm{CH}_{3}, J=7.1 \mathrm{~Hz}\right), 1.70-1.73(\mathrm{~m}, 4 \mathrm{H}$, tetralin $\left.\mathrm{CH}_{2}\right), 2.5\left(\mathrm{~m}, 4 \mathrm{H}\right.$, tetralin $\left.\mathrm{CH}_{2}\right), 4.08\left(\mathrm{q}, 2 \mathrm{H}, \mathrm{CH}_{2}, J=11.05 \mathrm{~Hz}\right), 7.15(\mathrm{~d}, 1 \mathrm{H}, \mathrm{CH}=, J=9.4 \mathrm{~Hz}), 7.40$ $(\mathrm{d}, 1 \mathrm{H}, \mathrm{CH}=, J=9.4 \mathrm{~Hz}), 6.97-7.4(\mathrm{~m}, 6 \mathrm{H}, \mathrm{Ar}-\mathrm{H}), 9.75(\mathrm{~s}, 1 \mathrm{H}, \mathrm{OH}) .{ }^{13} \mathrm{C}-\mathrm{NMR}: \delta 14.49\left(\mathrm{CH}_{3}\right), 22.2$ (tetralin $\left.\mathrm{CH}_{2}\right), 28.8$ (tetralin $\left.\mathrm{CH}_{2}\right), 125.7(\mathrm{CH}=)$ ) $147.2(\mathrm{CH}=), 111.8,113.3,115.6,123.7,126.2$, 128.4, 129.8, 136.7, 137.01, 141.9, 149.8, 153.2 (Ar-C), $188.56(\mathrm{C}=\mathrm{O}) . \mathrm{MS}, \mathrm{m} / \mathrm{z}$ (rel. int.): 174 (30), 166 (67), 159 (100), 137 (100), 131 (17). 


\subsection{1-Acetyl-4-(2,6-difluorophenyl)-3-(1,2,3,4-tetrahydronaphthalen-6-yl)-2-pyrazoline 4}

Hydrazine hydrate $(99 \%, 1.0 \mathrm{~mL})$ was added to a solution of compound $\mathbf{3 b}(2.98 \mathrm{~g}, 0.01 \mathrm{~mol})$ in acetic acid $(30 \mathrm{~mL})$ and the mixture was heated under reflux for $6 \mathrm{~h}$. and the excess solvent was distilled in vacuo. Cold water $(30 \mathrm{~mL})$ was then added to the remaining residue and the separated crude product was filtered, washed with water, dried and crystallized from acetic acid. IR: 2880-2910 (tetralin $\left.\mathrm{CH}_{2} \& \mathrm{CH}_{3}\right), 1710(\mathrm{C}=\mathrm{O}) .{ }^{1} \mathrm{H}-\mathrm{NMR}\left(\mathrm{DMSO}_{6}\right)$ ): $\delta$ 1.76-1.87 (m, 4H, tetralin $\left.\mathrm{CH}_{2}\right), 4.1$ (dd, $2 \mathrm{H}$, pyrazoline $\left.\mathrm{CH}_{2}, J=14.2 \mathrm{~Hz}\right), 2.15\left(\mathrm{~s}, 3 \mathrm{H}, \mathrm{CH}_{3}\right), 2.75-2.8\left(\mathrm{~m}, 4 \mathrm{H}\right.$, tetralin $\left.\mathrm{CH}_{2}\right), 6.5-6.9(\mathrm{~m}, 1 \mathrm{H}$, pyrazoline $\mathrm{CH}$ ), 7.0-7.5 (m, 6H, Ar-H). ${ }^{13} \mathrm{C}-\mathrm{NMR}: \delta 22.3$ (tetralin $\mathrm{CH}_{2}$ ), $24.4\left(\mathrm{CH}_{3}\right), 28.92$ (tetralin $\mathrm{CH}_{2}$ ), 38.67 (pyrazoline $\mathrm{CH}_{2}$ ), 39.67 (pyrazoline $\mathrm{CH}$ ), 111.8, 123.7, 126.2, 128.4, 129.8, 136.7, 137.01, 149.8, 141.9, 153.2 (Ar-C), 157.3 (imine C), 168.2 (C=O). MS m/z (rel. int.): 169 (72), 141 (40), $116(20), 96$ (46), 74 (100).

\subsection{4-Aryl-6-(1,2,3,4-tetrahydronaphthalen-6-yl)-1,2-dihydropyrimidine-2-thiones 5a,b}

A solution of potassium hydroxide $(1.7 \mathrm{~g}, 0.03 \mathrm{~mol})$ in water $(4 \mathrm{~mL})$ was added to a mixture of the appropriate $\alpha, \beta$-unsaturated ketone $3 \mathbf{a}, \mathbf{b}(0.01 \mathrm{~mol})$ and thiourea $(0.9 \mathrm{~g}, 0.012 \mathrm{~mol})$, in ethanol $(50 \mathrm{~mL})$ and the mixture was heated under reflux for $24 \mathrm{~h}$. The excess of solvent was then distilled off and the residue was acidified with dilute hydrochloric acid. The separated crude product was filtered, washed with water, dried and crystallized from aqueous ethanol.

4-(2,6-Dichlorophenyl)-6-(1,2,3,4-tetrahydronaphthalen-6-yl)-1,2-dihydropyrimidine-2-thione

(5a): IR: $3400(\mathrm{NH}), 2888-2921$ (tetralin $\left.\mathrm{CH}_{2}\right), 1050(\mathrm{C}=\mathrm{S}) .{ }^{1} \mathrm{H}-\mathrm{NMR}\left(\mathrm{DMSO}-\mathrm{d}_{6}\right): \delta$ 1.67-1.7 (m, 4H, tetralin $\left.\mathrm{CH}_{2}\right), 2.49-2.52\left(\mathrm{~m}, 4 \mathrm{H}\right.$, tetralin $\left.\mathrm{CH}_{2}\right), 3.55(\mathrm{~s}, 1 \mathrm{H}, \mathrm{NH}), 5.5(\mathrm{~s}, 1 \mathrm{H}$, pyrimidine $\mathrm{CH}), 6.6-7.1$ (m, 6H, Ar-H). MS, m/z (rel. int.): 159 (100), 131 (19), 91 (40), 78 (60).

4-(2,6-Difluorophenyl)-6-(1,2,3,4-tetrahydronaphthalen-6-yl)-1,2-dihydropyrimidine-2-thione

$(5 \mathbf{b})$ : IR: $3400(\mathrm{NH}), 2880-2925$ (tetralin $\left.\mathrm{CH}_{2}\right), 1050(\mathrm{C}=\mathrm{S}) .{ }^{1} \mathrm{H}-\mathrm{NMR}\left(\mathrm{DMSO}-\mathrm{d}_{6}\right): \delta 1.65-1.66(\mathrm{~m}, 4 \mathrm{H}$, tetralin $\left.\mathrm{CH}_{2}\right), 2.4-2.5\left(\mathrm{~m}, 4 \mathrm{H}\right.$, tetralin $\left.\mathrm{CH}_{2}\right), 3.5(\mathrm{~s}, 1 \mathrm{H}, \mathrm{NH}), 5.5(\mathrm{~s}, 1 \mathrm{H}$, pyrimidine $\mathrm{CH}), 6.57-7.14(\mathrm{~m}$, $6 \mathrm{H}, \mathrm{Ar}-\mathrm{H}$ ). ${ }^{13} \mathrm{C}-\mathrm{NMR}: \delta 22$ (tetralin $\mathrm{CH}_{2}$ ), 29 (tetralin $\mathrm{CH}_{2}$ ), 104 (pyrimidine $\mathrm{CH}$ ), 105.5, 111.2, 126.7, 129.6, 131.16, 134.26, 134.3, 135.9, 142.5, 157.3 (Ar-C), 185 (C=S). MS, m/z (rel. int.): 131 (8), 113 (2), $112(4), 96(31), 76(7)$.

\subsection{4-Aryl-6-(1,2,3,4-tetrahydronaphthalen-6-yl)-2-oxo-1,2-dihydropyridine-3-carbonitriles 6a,b}

A mixture of 6-acetyltetraline $1(1.74 \mathrm{~g}, 0.01 \mathrm{~mol})$, ethyl cyanoacetate $(1.13 \mathrm{~g}, 0.01 \mathrm{~mol})$, the appropriate aldehyde $\mathbf{2 a}, \mathbf{b}$, and ammonium acetate $(6.0 \mathrm{~g})$, was heated in $n$-butanol $(40 \mathrm{~mL})$ under reflux for $3 \mathrm{~h}$. On cooling, the separated yellow solid was filtered, washed with water and crystallized.

4-(2,6-Dichlorophenyl)-6-(1,2,3,4-tetrahydronaphthalen-6-yl)-2-oxo-1,2-dihydropyridine-3-carbonitrile (6a): IR: $3330(\mathrm{NH}), 2987-2910$ (tetralin $\left.\mathrm{CH}_{2}\right), 2228(\mathrm{CN}), 1616(\mathrm{C}=\mathrm{O}) .{ }^{1} \mathrm{H}-\mathrm{NMR}\left(\mathrm{CDCl}_{3}\right): \delta$ 1.79-1.82 (m, 4H, tetralin $\left.\mathrm{CH}_{2}\right), 2.76-2.82\left(\mathrm{~m}, 4 \mathrm{H}\right.$, tetralin $\left.\mathrm{CH}_{2}\right), 7.25-7.48(\mathrm{~m}, 7 \mathrm{H}, \mathrm{Ar}-\mathrm{H}), 7.75(\mathrm{~s}, 1 \mathrm{H}$, $\mathrm{NH}) .{ }^{13} \mathrm{C}-\mathrm{NMR}: \delta 22$ (tetralin $\left.\mathrm{CH}_{2}\right), 28$ (tetralin $\left.\mathrm{CH}_{2}\right), 114.4(\mathrm{CN}), 105.5,123.5,127.4,127.7,130.4$, 
132.7, 133.7, 127.8, 129.5, 138.4, 141.6, 151.5, 156.4, 165.5 (Ar-C \& pyridine-C), $162.69(\mathrm{C}=\mathrm{O})$. MS, m/z (rel. int.): 398 ( $\left.\mathrm{M}^{+}, 0.7\right), 205$ (100), 174 (4), 148 (3), 131 (11), 91 (20), 65 (3).

4-(2,6-Difluorophenyl)-6-(1,2,3,4-tetrahydronaphthalen-6-yl)-2-oxo-1,2-dihydropyridine-3-carbonitrile (6b): IR: $3430(\mathrm{NH}), 2990-2995$ (tetralin $\left.\mathrm{CH}_{2}\right), 2217(\mathrm{CN}), 1600(\mathrm{C}=\mathrm{O}) .{ }^{1} \mathrm{H}-\mathrm{NMR}\left(\mathrm{CDCl}_{3}\right): \delta$ 1.75-1.82 (m, $4 \mathrm{H}$, tetralin $\left.\mathrm{CH}_{2}\right), 2.76-2.81\left(\mathrm{~m}, 4 \mathrm{H}\right.$, tetralin $\left.\mathrm{CH}_{2}\right), 6.8-7.2(\mathrm{~m}, 7 \mathrm{H}, \mathrm{Ar}-\mathrm{H}), 7.61(\mathrm{~s}, 1 \mathrm{H}$, NH). MS, m/z (rel. int.): $362\left(\mathrm{M}^{+}, 0.5\right), 174$ (26), 166 (100), 159 (100), 131 (4), 118 (5).

\subsection{4-Aryl-6-(1,2,3,4-tetrahydronaphthalen-6-yl)-2-thioxo-1,2-dihydropyridine-3-carbonitriles 7a-c}

A mixture of 6-acetyltetraline $1(1.74 \mathrm{~g}, 0.01 \mathrm{~mol}), 2$-cyanothioacetamide $(1 \mathrm{~g}, 0.01 \mathrm{~mol})$, the appropriate aldehyde $\mathbf{2 a - b}$, and ammonium acetate $(6.0 \mathrm{~g})$, was heated under reflux in $n$-butanol $(40 \mathrm{~mL})$ for $3 \mathrm{~h}$. On cooling, the separated yellow solid was filtered, washed with water and crystallized.

4-(2,6-Dichlorophenyl)-6-(1,2,3,4-tetrahydronaphthalen-6-yl)-2-thioxo-1,2-dihydropyridine-3-carbonitrile (7a): IR: $3450(\mathrm{NH}), 2988-2995$ (tetralin $\mathrm{CH}_{2}$ ), $2240(\mathrm{CN}), 1340$ \& $1140(\mathrm{C}=\mathrm{S}) .{ }^{1} \mathrm{H}-\mathrm{NMR}$ (DMSO-d 6 ): $\delta$ 1.8-1.82 (m, 4H, tetralin $\left.\mathrm{CH}_{2}\right), 2.77-2.81\left(\mathrm{~m}, 4 \mathrm{H}\right.$, tetralin $\left.\mathrm{CH}_{2}\right), 6.5(\mathrm{~s}, 1 \mathrm{H}, \mathrm{NH}), 7.2-7.6(\mathrm{~m}, 7 \mathrm{H}$, Ar-H \& pyridine-H). ${ }^{13} \mathrm{C}-\mathrm{NMR}$ : $\delta 22$ (tetralin $\mathrm{CH}_{2}$ ), 30 (tetralin $\mathrm{CH}_{2}$ ), $115.5(\mathrm{CN}), 105,123.5,127.4$, 127.7, 130.4, 132.7, 133.7, 127.8, 129.5, 138.4, 141.6, 105.5, 151.5, 156.4 (Ar-C \& pyridine-C), 164 (C=S). MS, m/z (rel. int.): 281 (20), 207 (35), 147 (23), 135 (32).

4-(2,6-Difluorophenyl)-6-(1,2,3,4-tetrahydronaphthalen-6-yl)-2-thioxo-1,2-dihydropyridine-3-carbonitrile (7b): IR: $3450(\mathrm{NH}), 2910-2985$ (tetralin $\mathrm{CH}_{2}$ ), $2240(\mathrm{CN}), 1310$ \& $1090(\mathrm{C}=\mathrm{S}) .{ }^{1} \mathrm{H}-\mathrm{NMR}\left(\mathrm{DMSO}-\mathrm{d}_{6}\right)$ : $\delta$ 1.73-1.8 (m, 4H, tetralin $\left.\mathrm{CH}_{2}\right), 2.74-2.8\left(\mathrm{~m}, 4 \mathrm{H}\right.$, tetralin $\left.\mathrm{CH}_{2}\right), 5.18(\mathrm{~s}, 1 \mathrm{H}, \mathrm{NH}), 7.15-7.82(\mathrm{~m}, 7 \mathrm{H}$, Ar-H \& pyridine-H). MS, m/z (rel. int.): 378 (M+, 2.7), 361 (2), 207 (16), 159 (29), 78 (75).

4-(3-Ethoxy-4-hydroxyphenyl)-6-(1,2,3,4-tetrahydronaphthalen-6-yl)-2-thioxo-1,2-dihydropyridine-3carbonitrile (7c): IR: $3470(\mathrm{NH}), 3310(\mathrm{OH}), 2820-2995$ (tetralin $\left.\mathrm{CH}_{2}, \mathrm{CH}_{3}, \mathrm{CH}_{2}\right), 2250(\mathrm{CN}), 1310$ \& $1110(\mathrm{C}=\mathrm{S}) .{ }^{1} \mathrm{H}-\mathrm{NMR}\left(\mathrm{DMSO}_{6}\right)$ ) $\delta$ 1.33-1.35 (t, 3H, $\left.\mathrm{CH}_{3}, J=2.93 \mathrm{~Hz}\right), 1.73-1.8(\mathrm{~m}, 4 \mathrm{H}$, tetralin $\left.\mathrm{CH}_{2}\right), 2.76-2.8\left(\mathrm{~m}, 4 \mathrm{H}\right.$, tetralin $\left.\mathrm{CH}_{2}\right), 4.11\left(\mathrm{q}, 2 \mathrm{H}, \mathrm{CH}_{2} \mathrm{CH}_{3}, \mathrm{~J}=2.93 \mathrm{~Hz}\right), 9.7$ (s, $\left.1 \mathrm{H}, \mathrm{NH}\right), 7.0-7.6(\mathrm{~m}$, $7 \mathrm{H}, \mathrm{Ar}-\mathrm{H} \&$ pyridine-H). ${ }^{13} \mathrm{C}-\mathrm{NMR}: \delta 15.5\left(\mathrm{CH}_{3}\right), 22$ (tetralin $\left.\mathrm{CH}_{2}\right), 30$ (tetralin $\left.\mathrm{CH}_{2}\right), 58.5\left(\mathrm{CH}_{2} \mathrm{CH}_{3}\right)$, 115.5 (CN), 102.5, 104.0, 110.5, 129.4, 133.7, 135.4, 138.7, 140.7, 127.8, 128.5, 142.4, 145.6, 105.5, 153.5, 158.4, 166.5 (Ar-C \& pyridine-C), 168 (C=S). MS, m/z (rel. int.): 174 (33), 159 (100), 131 (20), $104(2)$.

\subsection{4-Aryl-6-(1,2,3,4-tetrahydronaphthalen-6-yl)-2-imino-1,2-dihydropyridine-3-carbonitriles 8a,b}

A mixture of 6-acetyltetraline $1(1.74 \mathrm{~g}, 0.01 \mathrm{~mol})$, malononitrile $(0.66 \mathrm{~g}, 0.01 \mathrm{~mol})$, the appropriate aldehyde $\mathbf{2 a - b}$, and ammonium acetate $(6.0 \mathrm{~g})$, was heated under reflux in $n$-butanol $(40 \mathrm{~mL})$ for $3 \mathrm{~h}$. On cooling, the separated yellow solid was filtered, washed with water and crystallized.

4-(2,6-Dichlorophenyl)-6-(1,2,3,4-tetrahydronaphthalen-6-yl)-2-imino-1,2-dihydropyridine-3-carbonitrile (8a): IR: 3500, $3450(\mathrm{NH}), 2820-2995$ (tetralin $\left.\mathrm{CH}_{2}\right), 2250(\mathrm{CN}) .{ }^{1} \mathrm{H}-\mathrm{NMR}\left(\mathrm{CDCl}_{3}\right): \delta$ 1.79-1.82 (m, 4H, tetralin $\left.\mathrm{CH}_{2}\right), 2.1(\mathrm{~s}, 1 \mathrm{H}, \mathrm{NH}), 2.81-2.83\left(\mathrm{~m}, 4 \mathrm{H}\right.$, tetralin $\left.\mathrm{CH}_{2}\right), 5.61(\mathrm{~s}, 1 \mathrm{H}, \mathrm{NH}), 7-7.6(\mathrm{~m}, 7 \mathrm{H}$, 
Ar-H \& pyridine-H). ${ }^{13} \mathrm{C}-\mathrm{NMR}: \delta 22.2$ (tetralin $\mathrm{CH}_{2}$ ), 28.7 (tetralin $\left.\mathrm{CH}_{2}\right), 114.9(\mathrm{CN}), 104.0123 .7$, 127.4, 127.6, 128.8, 130.01, 133.2, 136.8, 149.9, 159.06, 110.9, 128.6, 159.9, 162.0, 166.5 (Ar-C \& pyridine-C), $176.06(\boldsymbol{C}=\mathrm{NH}) . \mathrm{MS}, \mathrm{m} / \mathrm{z}$ (rel. int.): 207 (100), 159 (98), 151 (8), 131 (15), 119 (3).

4-(2,6-Difluorophenyl)-6-(1,2,3,4-tetrahydronaphthalen-6-yl)-2-imino-1,2-dihydropyridine-3-carbonitrile (8b): IR: 3470, $3450(\mathrm{NH}), 2820-2990$ (tetralin $\left.\mathrm{CH}_{2}\right), 2250(\mathrm{CN}) .{ }^{1} \mathrm{H}-\mathrm{NMR}\left(\mathrm{CDCl}_{3}\right): \delta 1.81-1.826(\mathrm{~m}$, 4H, tetralin $\left.\mathrm{CH}_{2}\right), 2.1(\mathrm{~s}, 1 \mathrm{H}, \mathrm{NH}), 2.81-2.83\left(\mathrm{~m}, 4 \mathrm{H}\right.$, tetralin $\left.\mathrm{CH}_{2}\right), 5.61(\mathrm{~s}, 1 \mathrm{H}, \mathrm{NH}), 6.9-7.67(\mathrm{~m}, 7 \mathrm{H}$, Ar-H \& pyridine-H). ${ }^{13} \mathrm{C}-\mathrm{NMR}: \delta 22.36$ (tetralin $\mathrm{CH}_{2}$ ), 28.91 (tetralin $\left.\mathrm{CH}_{2}\right), 115.8(\mathrm{CN}), 104.5$, 111.37, 111.68, 112.14, 123.98, 127.64, 129.15, 129.54, 131.03, 131.12, 137.23, 155.5, 1157.6, 161.3, 166.6 (Ar-C \& pyridine-C), 174.1 (C=NH). MS, m/z (rel. int.): 194 (5), 131 (100), 127 (100), 104 (100).

\subsection{Determination of the anticancer activity [26]}

Cell monolayers were fixed with $10 \%$ (w/v) trichloroacetic acid and stained for 30 minutes with $0.4 \%$ (wt/vol) sulforhodamine B (SRB) dissolved in 1\% acetic acid. Unbound dye was removed by four washes with $1 \%$ acetic acid, and protein-bound dye was extracted with $10 \mathrm{mM}$ unbuffered Tris base [tris (hydroxymethyl) aminomethane] for determination of optical density in a computer-interfaced, 96-well microtiter plate reader at $510 \mathrm{~nm}$.

\section{Conclusions}

New tetrahydronaphthaline derivatives bearing different heterocyclic moieties were tested for in vitro antitumor activity. The results indicated that the 2,6-dihaloarylchalcone derivatives $\mathbf{3 a}, \mathbf{b}$, the cyanopyridone derivatives $\mathbf{6 a}, \mathbf{b}$ and thioxopyridine derivatives $\mathbf{7 a - c}$ were the most effective against the cervix cell line (Hela), showing $\mathrm{IC}_{50}$ values of $3.5,10.5,7.1,10.9,8.1,5.9$ and $6.5 \mu \mathrm{g} / \mathrm{mL}$, respectively. All the tested compounds showed moderate to marginal activity against the breast carcinoma cell line MCF-7. Compound 3a was the most potent, with an $\mathrm{IC}_{50}$ value of $3.5 \mu \mathrm{g} / \mathrm{mL}$ against Hela and $4.5 \mu \mathrm{g} / \mathrm{mL}$ against MCF7 cell line. Compound 3a seems a promising new lead compound with a novel skeleton for further development towards a new potential clinical trials candidate.

\section{Acknowledgements}

The author would like to thank Abdelhamid Emwas, King Abdullah University for Science and Technology, Jeddah, Saudi Arabia, for recording the NMR spectra.

\section{References}

1. Balunas, M.J.; Kinghorn, A.D. Drug Discovery from medicinal plants. Life Sci. 2005, 78, 431-441.

2. Parkin, D.M. Global cancer statistics in the year 2000. Lancet Oncol. 2001, 2, 533-543.

3. Carmen, A.J.; Carlos, M. Medicinal Chemistry of Anticancer Drugs, 1st ed.; Elsevier: Amsterdam, The Netherlands, 2008; pp.1-8. 
4. Ferrante, A.; Augliera J.; Lewis, K. Cloning of an organic solvent-resistance gene in Escherichia coli: The unexpected role of alkylhydroperoxide reductase. Proc. Natl. Acad. Sci. USA 1995, 92, 7617-7621.

5. Hara, H.; Fujihashi, T.; Sakata, T. Tetrahydronaphthalene lignan compounds as potent anti-HIV type 1 agents. AIDS Res. Hum. Retroviruses 1997, 13, 695-705.

6. Ebeid, M.Y.; El-Zahar, M.I.; Kamel, M.M.; Omar, M.T.; Anwar, M.M. Novel 5,6,7,8tetrahydronaphth-2-ylheterocycles of possible biological activity. Egypt. Pharm. J. NRC 2004, 3, 49-65.

7. Nabih, I.; Zayed, A.; Kamel, M.M.; Motawie, M.S. Synthesis of new tetrahydronaphthyl-1,2,4triazines of potensial antimicrobial activity. Egypt. J. Chem. 1986, 29, 101-106.

8. Hussain, R.A.; Dickey, J.K.; Rosser, M.P.; Matson, J.A.; Kozlowski, M.R.; Brittain, R.J.; Webb, M.L.; Rose, P.M.; Fernandes, P.A. Novel class of non-peptidic endothelin antagonists isolated from the medicinal herb phyllanthus niruri. J. Nat. Prod. 1995, 58, 1515-1520.

9. Chalina, E.G.; Chakarova, L. Synthesis, hypotensive and antiarrhythmic activities of 3-alkyl-1-(2hydroxy-5,8-dimethoxy-1,2,3,4-tetrahydro-3-naphthalenyl)ureas or thioureas and their guanidine analogues. Eur. J. Med. Chem. 1998, 33, 975-983.

10. Kamel, M.M.; Michael, J.M. Synthesis and moulscicidal activity of some salicylamido tetralins. Egypt. J. Bilharziasis 1988, 10, 121-125.

11. Cimetière, B.; Dubuffet, T.; Muller, O.; Descombes, J.; Simonet, S.; Laubie, M.; Verbeuren, T.J.; Lavielle, G. Synthesis and biological evaluation of new tetrahydronaphthalene derivatives as thromboxane receptor antagonists. Bioorg. Med. Chem. Lett. 1998, 8, 1375-1340.

12. Takami, M.; Tsukada, W. Effect of DP-1904, a thromboxane synthetase inhibitor, on antigen- and spasmogen-induced bronchoconstriction in rodents. Eur. J. Pharmacol. 1999, 366, 253-259.

13. Rogóz, Z.; Skuza, G.; Kodziñska, A. Anxiolytic-and antidepressant-like effects of 7-OH DPAT, preferential dopamine D3 receptor agonist in rats. Pol. J. Pharmacol. 2004, 56, 519-529.

14. Kitamura, Y.; Araki, H.; Shibata, K.; Gomita, Y.; Tanizaki, Y. 5-HT $1 \mathrm{~A}$ receptor full agonist, 8-OH-DPAT, exerts antidepressant-like effects in the forced swim test in ACTH-treated rats. Eur. J. Pharmacol. 2003, 481, 75-77.

15. Amin, K.M.; El-Zahar, M.I.; Anwar, M.; Kamel, M.; Mohamad, M. Synthesis and anticancer activity of novel tetralin-6-yl pyridine and tetralin-6-yl pyrimidine derivatives. Acta Pol. Pharm. 2009, 66, 279-291.

16. Dong, Y.Z.; Shi, Q.; Nakagawa-Goto, K.; Wu, P.C.; Bastow, K.F.; Morris-Natschke, S.L.; Lee, K.H. Antitumor agents 269. Non-aromatic ring-A neotanshinlactone analog, TNO, as a new class of potent antitumor agents. Bioorg. Med. Chem. Lett. 2009, 19, 6289-6292.

17. Öztürk, G.; Erol, D.D.; Uzbay, T.; Aytemir, M.D. Synthesis of 4(1H)-pyridinone derivatives and investigation of analgesic and antiinflammatory activities. Farmaco 2001, 56, 251-256.

18. Findlay, J.A.; Tam, W.H.J.; Krepinsky, J. The chemistry of some 6-methyl-4-hydroxy-2-pyridones. Can. J. Chem. 1978, 56, 613-616.

19. Abadi, A.; Al-Deeb, O.; Al-Afify, A.; El-Kashef, H. Synthesis of 4-alkyl (aryl)-6-aryl-3-cyano$2(1 H)$-pyridinones and their 2-imino isosteres as nonsteroidal cardiotonic agents. Farmaco 1999, 54, 195-201. 
20. Storck, P.; Aubertin, A.; Grierson, D.S. Tosylation/mesylation of 4-hydroxy-3-nitro-2-pyridinones as an activation step in the construction of dihydropyrido[3,4-b] benzo[f][1,4]thiazepin-1-one based anti-HIV agents. Tetrahedron Lett. 2005, 46, 2919-2922.

21. Macdonald, G.E.; Puri, A.; Shilling, D.G. Interactive effect of photoperiod and fluridone on growth, reproduction, and biochemistry of dioecious hydrilla (Hydrilla Verticillata). Weed Sci. 2008, 56, 189-195.

22. Evidente, A.; Fiore, M.; Bruno, G.; Sparapano, L.; Motta, A. Chemical and biological characterisation of sapinopyridione, a phytotoxic 3,3,6-trisubstituted-2,4-pyridione produced by Sphaeropsis sapinea, a toxigenic pathogen of native and exotic conifers, and its derivatives. Phytochemistry 2006, 67, 1019-1028.

23. Cocco, M.T.; Congiu, C.; Onnis, V. Synthesis and antitumour activity of 4-hydroxy-2-pyridone derivatives. Eur. J. Med. Chem. 2000, 35, 545-552.

24. Cocco, M.T.; Congiu, C.; Onnis, V. New bis(pyridyl)methane derivatives from 4-hydroxy-2pyridones: synthesis and antitumoral activity. Eur. J. Med. Chem. 2003, 38, 37-47.

25. Allinger, N.L.; Jones, E.S. Synthesis of some functionally substituted benzocyclanones. J. Org. Chem. 1962, 27, 70-76.

26. Storeng, P.; Ritsa, S.; Scudiero, D.; Monks, A.; McMahon, J.; Vistica, D.; Warren, J.T.; Bokesch, H.; Kenney, S.; Boyd, M.R. Anticancer-drug screening. J. Natl. Cancer Inst. 1990, 82, 1107-1112.

27. Ghoshal, K.; Jacob, S.T. An alternative molecular mechanism of action of 5-fluorouracil, a potent anticancer drug. Biochem. Pharmacol. 1997, 53, 1569-1575.

Sample Availability: Contact the author.

(C) 2011 by the authors; licensee MDPI, Basel, Switzerland. This article is an open-access article distributed under the terms and conditions of the Creative Commons Attribution license (http://creativecommons.org/licenses/by/3.0/). 